\section{Role of pulmonary ultrasound in the diagnosis of acute respiratory failure}

\author{
Marcello Romano, ${ }^{1}$ Roberto Risicato, ${ }^{2}$ \\ Enzo Vicari, ${ }^{3}$ Giulia Romano ${ }^{4}$ \\ ${ }^{1}$ Geriatrics Department, Garibaldi \\ Hospital, Catania; ${ }^{2}$ Internal Medicine \\ Department, Muscatello Hospital, \\ Augusta (SR); ${ }^{3}$ Direction of Geriatrics \\ School, University of Catania; \\ ${ }^{4}$ Department of Clinical and \\ Experimental Medicine, Internal \\ Medicine, University of Catania, Italy
}

\begin{abstract}
Dyspnea is the main clinical manifestation of acute respiratory failure; however, it can be induced by different causes that require differential diagnosis. In elderly patients, the medical examination is limited by cognitive and physical deficits and the genesis of dyspnea is often multifactorial; therefore, the definition of the causes is more complex. In recent years, among the various diagnostic methods, pulmonary ultrasonography has found increasing interest and the main patterns of ultrasound semeiotics have been described, specially in the emergency and critical care setting, as for pneumonia, pleural effusion, pneumothorax, pulmonary infarction, interstitial pathology. The most recent Literature emphasizes the role of multi-organ ultrasound (pleuro-pulmonar; cardiac; caval and iliac-femoral venous), to diagnose with good accuracy the causes of dyspnea. In this review we report the lung ultrasound semeiotic patterns, useful for the bedside or pointof-care detection of the cause of dyspnea, particularly respiratory and/or cardiac, during the medical examination.
\end{abstract}

\section{Introduction}

Dyspnea is the main symptom in the patient with acute respiratory failure. However, it requires a differential diagnosis process because it can be induced by different possible causes: i) respiratory (tracheal, bronchial, pulmonary, pleural); ii) ab extrinsic compression (pleural effusion, endothorax masses, hiatal hernia); iii) musculoskeletal (trauma, neuromuscular diseases, obesity, sarcopenia); iv) cardiac; v) metabolic (acidosis); vi) haematological (anemia, thoracic masses); vii) psychogenic.
Specifically in elderly patients the genesis of dyspnea is often multifactorial, due to frequent comorbidities, making the diagnostic and therapeutic decisions more difficult.

Ultrasound presents two main strengths: i) non-invasive nature of surface applications, practically free of iatrogenic risk; ii) bedside or point-of-care use, for example in the ward or at home, thanks to the availability of transportable equipment, suitable for the logistical problems of frail patients. ${ }^{1}$

According to the Health Technology Assessment (HTA) statements, the validated contexts of chest ultrasound are: a reliable complementary diagnostic tool in the study of pleural effusions and guidance to thoracentesis; detection but not characterization of the lesions adhering to the pleura; study of lymph nodes and chest wall lesions and as a guide to biopsy. ${ }^{2}$ However, despite this limitation, the bedside ultrasound of some body districts (such as chest, heart, caval and femoral veins) can provide important information to guide, confirm or rule out clinical diagnosis by integrating the patient's physical examination.

Below we report the main patterns of pulmonary ultrasound semeiotics, described in Literature and useful for the bedside or point-of-care detection of the cause of dyspnea, particularly respiratory and/or cardiac.

Ultrasound is not adequate for studying the normal lung, mainly due to high absorption coefficient of the air; however, it acquires importance in the presence of alterations of pleura or subpleural lung, such as pleural effusion, lung consolidations of various type (lobar or segmental pneumonia, pulmonary infarction, benign or malignant neoplasms, atelectasis), interstitial lung diseases, pulmonary edema, pneumothorax. Moreover, although the ultrasound meets the obstacle of the thoracic bones (rib, scapula, sternum, clavicle), the intercostal approach of the probe allows the exploration of about $70 \%$ of the pleuro-pulmonary surface. The examination is performed with an ultrasound machine equipped with a convex probe, using intermediate frequency values $(3.5-5 \mathrm{MHz})$, that allows a reasonable resolution of both the pleural line and the subpleural space, maintaining an overview of the thoracic section; the use of high frequency probes (over 8 $\mathrm{MHz}$ ) is useful for the evaluation of the most superficial structures, such as the chest wall. ${ }^{3-5}$ The scanner is preset for use with a convex probe, a frequency of $3.5-5 \mathrm{MHz}$, a scanning depth of 7-15 cm and a gain of $55 \%,{ }^{6}$ initially focusing the ultrasound beam just below the pleural line; the usefulness of the harmonic ultrasound of the lung has no clear evidence in the literature.

The examination is performed with
Correspondence: Marcello Romano,

Geriatrics Department, Garibaldi Hospital, Via Palermo 636, 95100 Catania, Italy.

Tel.:/Fax: +39 0957593941

E-mail: marcelloromano@tin.it

Key words: Ultrasonography; lung; respiratory failure.

Received for publication: 1 August 2018.

Revision received: 22 October 2018

Accepted for publication: 25 October 2018.

This work is licensed under a Creative

Commons Attribution-NonCommercial 4.0

International License (CC BY-NC 4.0).

(C) Copyright M. Romano et al., 2018

Licensee PAGEPress, Italy

Geriatric Care 2018; 4:7730

doi:10.4081/gc.2018.7730

multiple longitudinal and intercostal scans, following predetermined procedures or starting from the sites of altered thoracic physical examination. ${ }^{7}$

The ultrasound semeiotics of the pleuro-pulmonary pathology has been described in recent decades, supporting a growing use of the ultrasonography to define the diagnosis in dyspnoic patients, with good diagnostic accuracy in the emergency and critical care setting, ${ }^{8}$ although the debate is still open on the distinction between different forms of consolidation as well as between different forms of interstitial alterations. ${ }^{9}$

\section{Pneumonia}

When pneumonia has a peripheral, subpleural extension, it is placed in the space that can be explored by ultrasound ${ }^{10}$ and literature suggests that most pneumonias in critically ill patients is in contact with the pleura. ${ }^{11}$ In these patients pneumonia has an ultrasonographic pattern dense of the parenchyma, often hypoechoic, which shows an echo-texture similar to that of liver or spleen (tissue-like pattern); within the pulmonary consolidation it is possible to detect hyperechoic linear striae, which can be referred to air in intraparenchymal bronchial branches (air bronchograms) (Figure 1). ${ }^{10,12-14}$ Although this aspect is not pathognomonic, a recent meta-analysis of studies in critical or emergency areas (1.172 patients) reported a sensitivity and specificity of ultrasound for pneumonia of $94 \%$ and $96 \%$, respectively. ${ }^{15}$ In particular, a study conducted among multimorbid patients admitted to an acute geriatric ward, lung ultrasound was more accurate than chest $\mathrm{X}$ - 
ray for the diagnosis of pneumonia (90 vs $77 \%$ ), particularly in those with frailty. ${ }^{16}$

\section{Polmonary infarction}

Pulmonary infarction appears as an hypoechoic consolidation area without vascular signals, frequently triangular or wedge-shaped with sub-pleural base (Figure 2); in our experience this aspect is not specific, but the diagnosis of pulmonary infarction is suggestive if there is ultrasound evidence of deep venous thrombosis along the iliac-femoral axis and dilatation of the right ventricle. ${ }^{17,18}$

\section{Neoplasms}

Sub-pleural neoplasms, primitive or secondary, appear as solid round areas, usually hypoechoic, single or multiple, with an irregular profile, demarcated by the aerated lung, with a variable level of vascularization, if it is evaluable in a dyspnoic patient, usually poor in metastases ${ }^{4}$ (Figure 3A-C).

\section{Interstitial alterations}

The so-called B-lines are an artifact (ring down artifact) due to resonance of the ultrasound beam, caused by interfaces made of fluid (exudate or transudate) and air. They consist of mobile hyperechoic lines that originate from the pleuro-parenchymal plane and distally extend from it; their presence indicates interstitial alterations and their number would increase with decreasing air content and increasing lung density. ${ }^{19,20}$
B-lines, however, are not a very specific echographic sign; indeed, they can be associated with different interstitial alterations such as: pulmonary edema, pulmonary fibrosis, lung contusions, pneumonia or acute respiratory distress syndrome (ARDS); moreover, single B-lines can be observed even in healthy subjects and in those who had undergone pneumonectomy. ${ }^{6,20}$ Despite this limitation, the clinical integration of B-line detection with anamnestic and physical findings (e.g. fever, chest pain, trauma, cough, hemoptysis, etc.) can supports diagnostic and therapeutic decision-making. ${ }^{20}$

Some studies have reported the usefulness of B-lines to distinguish cardiogenic pulmonary edema (in which the B-lines would be numerous, widespread and bilateral, up to the white lung pattern) from other interstitial pulmonary alterations, such as pulmonary fibrosis or interstitial pneumonia (in which the B-lines would be less numerous and irregularly or focally distributed) (Figure 4A-C); this argument has not been confirmed in other studies and is still debated. 6,9

\section{Pleural effusion}

The presence of pleural effusion is indicated by a mainly anechoic area that separates the thoracic wall or the diaphragm from the adjacent lung, which appears atelectasic according to the extent of the effusion (Figure 5A and B). Especially in elderly bedridden patients, ultrasound has better diagnostic accuracy than chest X-ray. ${ }^{21}$

Ultrasonography can detect even small effusions (about $100 \mathrm{~mL}$ ) and allows the volumetric evaluation of pleural effusion. One of the different methods proposed is based on the following formula: Volume $(m L)=16 \times$ parietal to visceral pleura distance $(\mathrm{mm})$ at the mid-diaphragm..$^{22}$ In clinical practice, however, a qualitative assess-

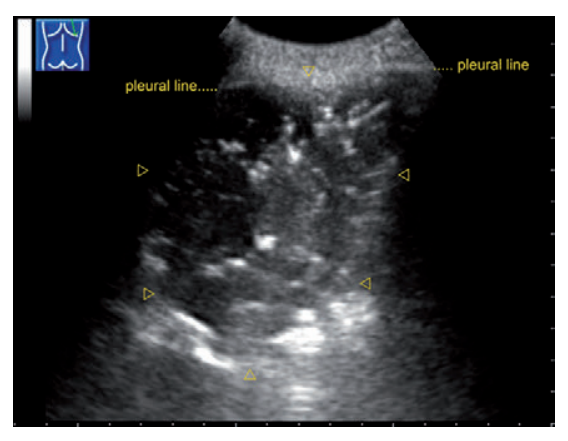

Figure 1. Pneumonia - Extended consolidation area (arrowheads), with liver-like echotexture and multiple hyperechoic striae (compatible with air bronchograms).

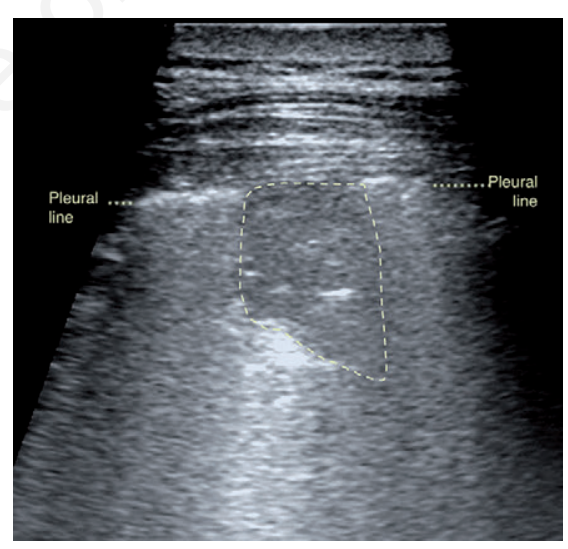

Figure 2. Pulmonary infarction - Area of consolidation (dotted line), hypoechoic, wedgeshaped, with disappearance of the pleural line; this pattern appears poorly differentiable from that of segmental pneumonia.
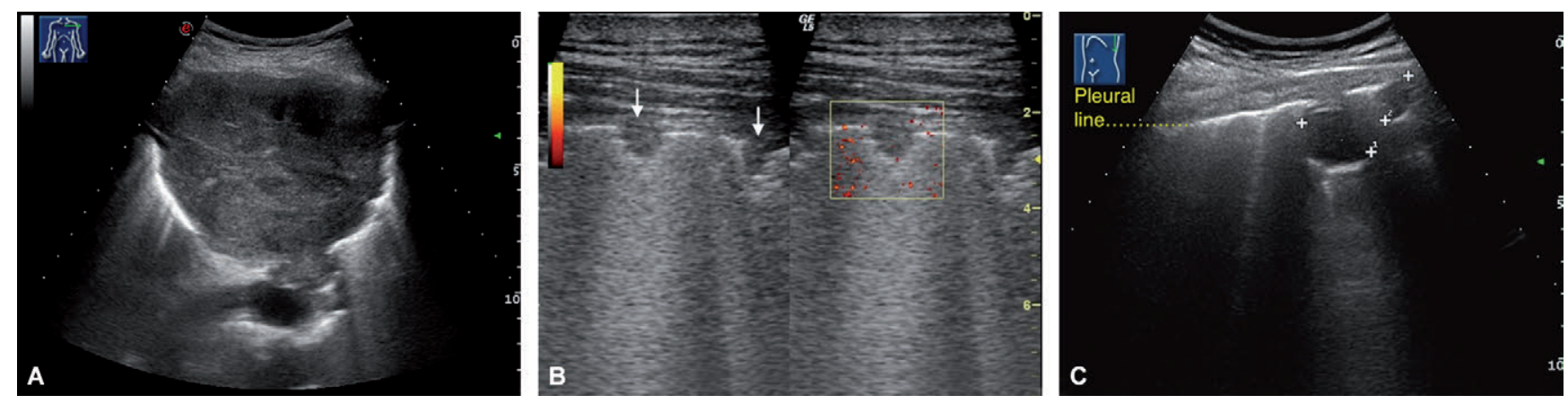

Figure 3. A) Pulmonary adenocarcinoma - A solid, hypoechoic, roundish expanding process that displaces the adjacent pulmonary parenchyma; inside some nuanced anechoic area, referable to colliquative necrosis; absent hyperechoic striae; disappearance of the pleural line. B) Sub-pleural pulmonary metastases (breast cancer) - Two small subpleural hypoechoic nodules (arrows); the echo-color shows no vascular signals. C) Sub-pleural pulmonary metastases (bepatocarcinoma) - Two hypo-anechoic nodules (calipers) that interrupt the pleural line. 
ment of the effusion volume is usual, distinguishing categories such as: minimal, small, moderate, large.

In the context of effusion, the presence of mobile echoes may indicate exudate, pus or blood, while the presence of septa indicates its multiloculated structure (Figure 5C). Moreover, being an imaging method in real time, the ultrasound also easily supports the pleural drainage procedures, allowing visualizing the needle during the introduction or, at least, to define previously the route and the depth of introduction, thus reducing complications from accidental puncture of the lung or other structures. ${ }^{4}$

\section{Pneumothorax}

In patients with pneumothorax, ultrasound shows the presence of static A-lines, which are an artifact due to the presence of pleural air that generates multiple reflections from the chest wall.

In the case of pneumothorax, $A$-lines typ- ically appear static, because they do not follow the respiratory movement of the lung, displaced from the parietal pleura (absence of pleural sliding); in fact, they represent extra-pulmonary air in the pleural cavity.

In the supine patient, due to the effect of gravity, these reverberations can be detected in the parasternal region around the II-IIIIV intercostal space. The diagnosis of pneumothorax is confirmed by the finding of the lung point, that is the borderline between the pleural air and the lung ${ }^{19}$ (Figure 6). In the setting of emergencies and traumas, ultrasound appears to have a high accuracy, better than portable chest $\mathrm{x}$-ray, to detect pneumothorax. $^{23}$

\section{Multi-organ ultrasonography}

More recently, the literature has reported the diagnostic utility of multi-organ ultrasonography to establish the cause of dyspnea with good diagnostic accuracy: not only pleuro-pulmonary, but also at the same time cardiac, caval and femoral venous ultrasound. ${ }^{17}$ Thus, for example, the presence of right ventricular dilatation and femoral venous thrombosis orients towards venous thromboembolism, while dilatation of the vena cava with poor respiratory excursion of the caliber indicates cardiac failure (Figure 7), which can be confirmed by the presence of ventricular abnormalities (dilatation, hypertrophy, hypokinesia). Specifically, a good correlation between the diameter of the inferior vena cava and right atrial pressure is reported in the Literature. ${ }^{24}$

\section{Conclusions}

The role of ultrasound for the diagnostic definition of patients with dyspnea was mainly developed in the critical and emergency area, ${ }^{11,19,23,25}$ but it is still a topic of debate in other points-of-care, such as geriatrics. This is because pulmonary ultrasound appears to be a sensitive method for detection of sub-pleural consolidations and
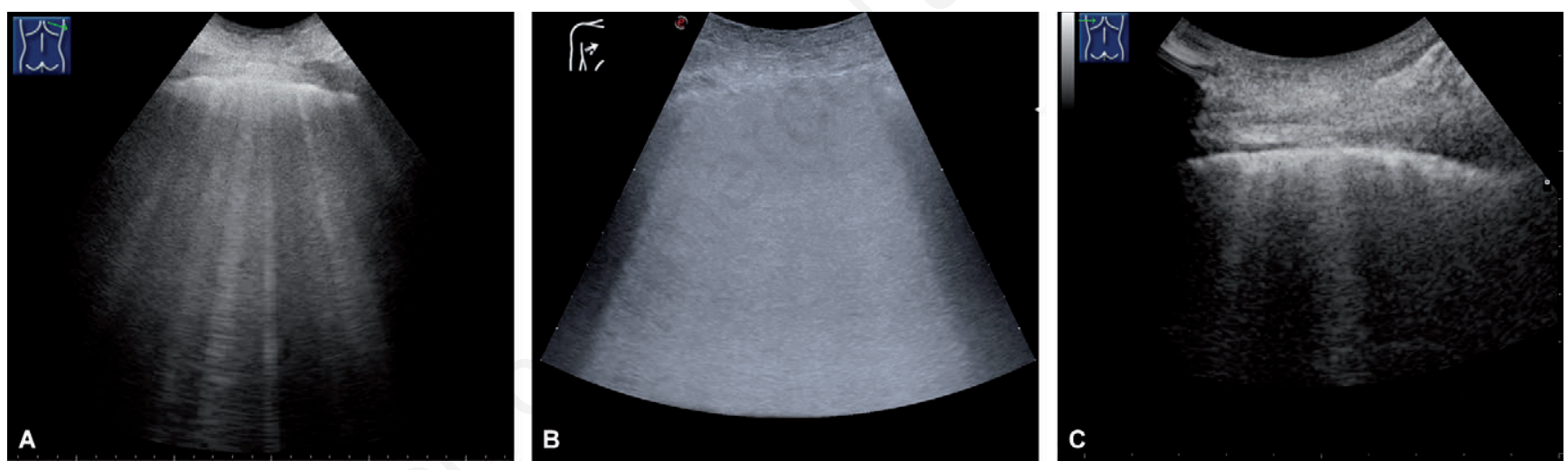

Figure 4. A) Acute pulmonary edema - Multiple B-lines, vertical, originating from the pleural plane and extending distally. B) Acute pulmonary edema - White lung pattern. C) Pulmonary fibrosis - Thickened and irregular pleuro-pulmonary profile; presence of irregularly distributed B-lines.
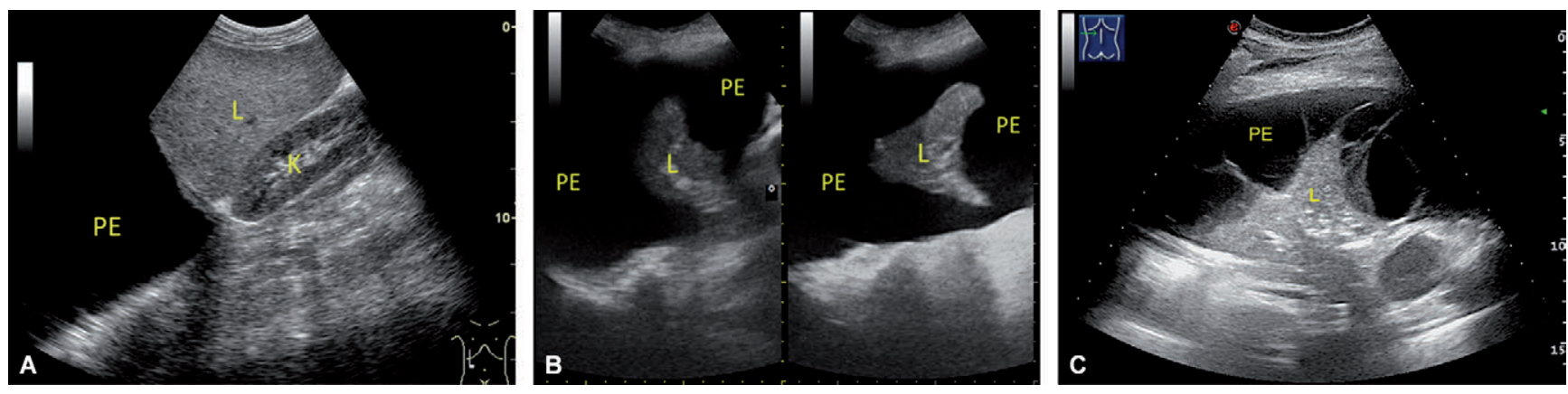

Figure 5. A) Pleural effusion (PE) - Large anechoic area overlying the diaphragm (L, liver; K, kidney). B) PE - Transverse and longitudinal scan of the right thorax: diffuse anechoic area that surrounds and compresses the lung (L), atelectasic. C) Multiloculated PE Large hypo-anechoic area surrounding atelectatic lung $(\mathrm{L})$; present numerous echogenic septa between parietal and pulmonary pleura. 


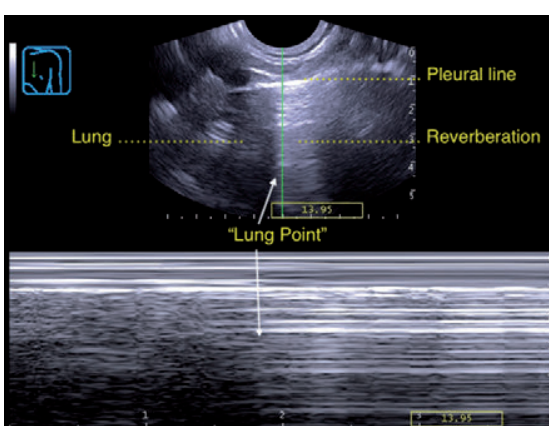

Figure 6. Pneumothorax - B-mode ultrasound (above): hyperechoic reverberation band (A-Lines) originating from the pleural line and distally directed (static in real time imaging); M-Mode ultrasound (below): on the right bar code sign, corresponding to the static reverberations of the pneumothorax; the lung point represents the borderline between the lung and the pleural air.

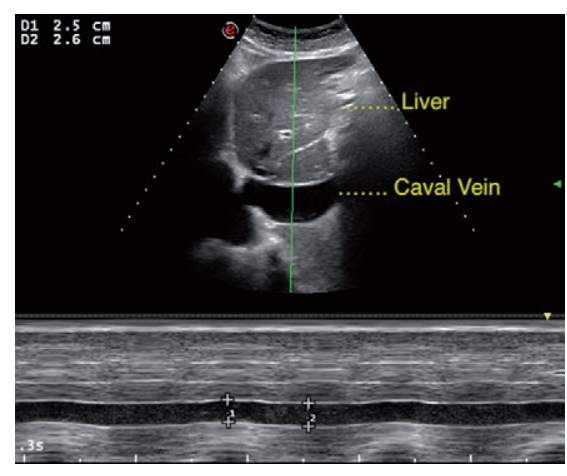

Figure 7. Heart failure - Inferior vena cava in longitudinal scan: B-mode (above) and $M$ Mode (below); the caliber of the vein is dilated $(2.5 \mathrm{~cm})$ without respiratory changes.

for interstitial alterations (B-lines), but if used alone, in both cases it does not allow their characterization.

We consider reasonable that in any care setting the pulmonary or, better, multi-organ ultrasound is most useful if the ultrasound findings are evaluated in the patient's clinical context, ie considering the presence or absence of other signs and symptoms, such as fever, pain, cyanosis, cough, hemoptysis, jugular vein dilatation, hypotension, edema, etc.

In this perspective, bedside or point-ofcare ultrasound could be a useful decisionmaking tool, during the medical examination, to make the diagnosis faster and more correct, so the therapy more timely and appropriate.

\section{References}

1. Romano M. L'ecografia nel paziente anziano o cronico. Atti Congresso Nazionale ASSIMEFAC "Il Paziente Cronico e la Continuità delle cure", Roma, 22-24 Ottobre 2009, pp 54-55. Available from: http://www.medinterna.net/wp-content/uploads/2015/12/ romanoecografiaanzianoassimefac09.pdf

2. Gizewski ER, Forsting M, Krombach GA, Schöffski O. Health Technology Assessment (HTA) Entwicklung im Gesundheitswesen und Potenzial in der Radiologie. Radiologe 2014;54: 589-98.

3. Romano M, Romano G, Romano R, et al. Formazione dell'immagine ed artefatti in ecografia. In: Schiavone C, Romano M, eds. Manuale italiano di ecografia internistica. Roma: Delfino editore; 2013. pp 25-40.

4. Sperandeo M, Sperandeo G, Piattelli ML. Ecografia del torace. In: Schiavone C, Romano M, eds. Manuale italiano di ecografia internistica. Roma: Delfino editore; 2013. pp 659-670.

5. Gargani L, Volpicelli G. How I do it: Lung ultrasound. Cardiovascular Ultrasound 2014;12:25.

6. Sperandeo M, Varriale A, Sperandeo G, et al. Characterization of the normal pulmonary surface and pneumonectomy space by reflected ultrasound. J Ultrasound 2011;14:22-7.

7. Volpicelli G, Mussa A, Garofalo G, et al. Bedside lung ultrasound in the assessment of alveolar-interstitial syndrome. Am J Emerg Med 2006;24:689-96.

8. Volpicelli G. Point-of-care lung ultrasound. Praxis 2014;103:711-6.

9. Hew M, Tay TR. The efficacy of bedside chest ultrasound: from accuracy to outcomes. Eur Respir Rev 2016;25:230-46.

10. Blaivas M. Lung Ultrasound in evaluation of pneumonia. J Ultrasound Med 2012;31:823-6.

11. Lichtenstein DA. BLUE-protocol and FALLS-protocol: two applications of lung ultrasound in the critically ill. Chest 2015;147:1659-70.

12. Weinberg B, Diakoumakis EE, Kass EG, et al. Air bronchogram: sonographic demonstration. AJR Am J Roentgenol 1986;147:593-5.

13. Lichtenstein D, Meziere G, Seitz J. The dynamic air bronchogram. A lung ultrasound sign of alveolar consolidation ruling out atelectasis. Chest 2009; 135:1421-5.
14. Cortellaro F, Colombo S, Coen D, Duca PG. Lung ultrasound is an accurate diagnostic tool for the diagnosis of pneumonia in the emergency department. Emerg Med J 2012;29:19-23.

15. Chavez MA, Shams N, Ellington LE, et al. Lung ultrasound for the diagnosis of pneumonia in adults: a systematic review and meta-analysis. Respir Res 2014;15:50.

16. Ticinesi A, Lauretani F, Nouvenne A, et al. Lung ultrasound and chest $\mathrm{x}$-ray for detecting pneumonia in an acute geriatric ward. Medicine 2016;95:27.

17. Nazerian P, Vanni P, Volpicelli G, et al. Accuracy of point-of-care multiorgan ultrasonography for the diagnosis of pulmonary embolism. Chest 2014;145: 950-95.

18. Niemann T, Egelhof T, Bongartz G. Transthoracic sonography for the detection of pulmonary embolism - a metaanalysis. Ultraschall Med 2009;30:150-6.

19. Volpicelli G, Elbarbary M, Blaivas M, et al. International evidence based recommendations for point-of-care lung ultrasound. Intensive Care Med 2012; 38:577-91.

20. Dietrich CF, Mathis G, Blaivas M, et al. Lung B-line artefacts and their use. J Thorac Dis 2016;8:1356-65.

21. Romano G, Cappello A, Perracchio G, et al. Diagnostic accuracy of bedside abdominal ultrasonography and chest X-ray for pleural effusion in elderly bedridden patients. Intern Emerg Med 2012;7:S476-7.

22. Usta E, Mustafi M, Ziemer G. Ultrasound estimation of volume of postoperative pleural effusion in cardiac surgery patients. Interact Cardiovasc Thorac Surg 2010;10:204-7.

23. Wongwaisayawan S, Suwannanon R, Sawatmongkorngul S, Kaewlai R. Emergency Thoracic US: The Essentials. Radiographics 2016;36:640-59.

24. Otto CM. Echocardiographic evaluation of left and right ventricular systolic function. In: Otto CM, ed. Textbook of clinical echocardiography. 2nd ed. Philadelphia: WB Saunders; 2000. p 120-1.

25. Lichtenstein DA, Mezière GA. Relevance of lung ultrasound in the diagnosis of acute respiratory failure: the BLUE protocol. Chest 2008;134:117-25. Erratum in: Chest 2013;144:721. 